\title{
BEAM LOSS, RESIDUAL RADIATION, AND COLLIMATION AND SHIELDING IN THE FERMILAB BOOSTER*
}

\author{
A. Drozhdin ${ }^{\dagger}$, P. Kasper, O. Krivosheev, J. Lackey, \\ N. Mokhov, M. Popovic, R. Webber, FNAL, Batavia, IL 60510, USA
}

\section{Abstract}

During its 30 years of operation, the Fermilab Booster has served only as an injector for the relatively low repetition rate proton accelerator complex. With the construction of an $8 \mathrm{GeV}$ target station for the $5 \mathrm{~Hz}$ MiniBooNE neutrino beam and rapid multi-batch injection into the Main Injector for the NuMI experiment, the demand for Booster protons will increase dramatically over the next few years. This implies serious constraints on beam losses in the machine. A collimation system and shielding design based on realistic Monte Carlo simulations are presented. A two-stage beam collimation system with local shielding has been designed. It provides adequate protection of the Booster components and environment by localizing operational losses. This loss control is a key to the entire future Fermilab high energy physics program.

\section{COLLIMATION SYSTEM}

There are 24 identical cells in the Booster lattice each with a six-meter long straight section. Nine of the long straight sections are occupied by RF cavities, four by extraction kicker and septum magnets, and one by the injection system. The rest can be used for a beam halo collimation. It was proposed to use straight sections number 6 and 7 for collimation because this region is far from the engineering, support and office buildings.

In this study, multi-turn particle tracking in the accelerator and simulation of halo interactions with the collimators are done with the STRUCT [1] code. Full-scale Monte Carlo hadronic and electromagnetic shower simulations in the lattice elements, shielding, tunnel and surrounding soil are done with MARS14 [2] code.

The beam power at the top accelerator energy with $15 \mathrm{~Hz}$ repetition rate and $5 \cdot 10^{12} \mathrm{ppp}$ is $0.1 \mathrm{MW}$. Assuming that $30 \%$ of the beam is lost at injection $\left(E_{k i n}=0.4 \mathrm{GeV}\right)$ and $2 \%$ at the top accelerator energy $\left(E_{k i n}=8 \mathrm{GeV}\right), 1.44 \mathrm{~kW}$ and $1.92 \mathrm{~kW}$ of beam loss are distributed around the ring with a beam loss rate of $20-90 \mathrm{~W} / \mathrm{m}$ in the RF cavities. This level is two orders of magnitude higher than can be accepted in the accelerator. The purpose of the beam halo cleaning system is to localize proton losses in a specially shielded short section, thus to reduce irradiation of the rest of the machine to the acceptable levels.

A 2-stage collimation system has been proposed with $0.1 \mathrm{~mm}$ thick tungsten horizontal and vertical primary collimators followed by four secondary ones (Fig. 1, Table 1).

\footnotetext{
* Work supported by the Universities Research Association, Inc., under contract DE-AC02-76CH03000 with the U. S. Department of Energy.

† drozhdin@fnal.gov
}
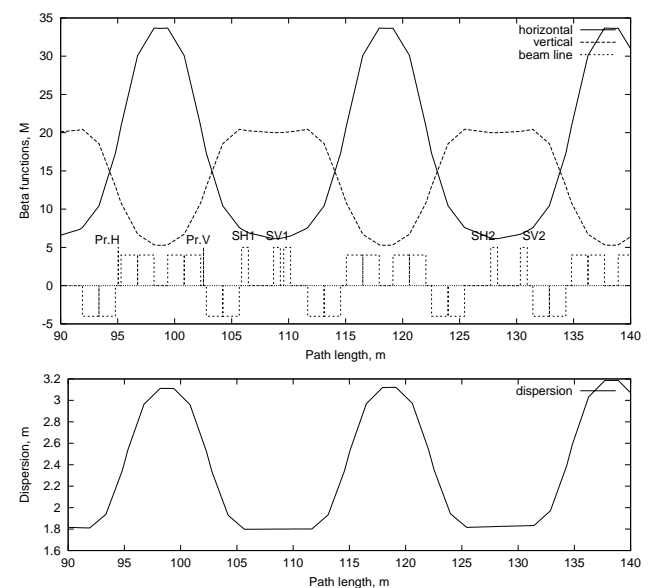

Figure 1: Beta functions and dispersion in the collimation region.

Primary collimators are placed at the edge of the circulating beam after injection. Secondary collimators are positioned with $0.5 \sigma$ offset with respect to the primary ones at phase advances that are optimal to intercept most of particles outscattered from the primary collimators during the first turn after the halo interaction with the primary collimators.

Table 1: $\beta$-functions, dispersion and phase advances between collimators.

\begin{tabular}{|c|c|c|c|c|}
\hline element & $\begin{array}{c}\beta \text {-functions } \\
\mathrm{m}\end{array}$ & $\begin{array}{c}\text { disper- } \\
\text { sion } \\
\mathrm{m}\end{array}$ & \multicolumn{2}{|c|}{$\begin{array}{c}\text { Phase advance between } \\
\text { primary and secondary } \\
\text { collimators, degree }\end{array}$} \\
\hline & hor. / ver. & & horizontal & vertical \\
\hline horiz. prim. PrH. & $19.0 / 11.9$ & 2.44 & 0 & - \\
vert. prima. PrV. & $19.0 / 11.9$ & 2.43 & - & 0 \\
secondary SH1 & $7.4 / 20.4$ & 1.80 & 37 & - \\
secondary SV1 & $6.2 / 20.0$ & 1.80 & - & 20 \\
secondary SH2 & $6.2 / 20.0$ & 1.82 & 154 & - \\
secondary SV2 & $7.1 / 20.3$ & 1.83 & - & 127 \\
\hline
\end{tabular}

A $0.15 \mathrm{~mm}$ thick graphite primary collimator is optimal for injection, but unfortunately this thickness is not enough for the top energy. An optimal primary collimator at the top energy would be $0.2 \mathrm{~mm}$ tungsten collimator. Horizontal phase space at injection and at the top energy in the primary and secondary collimators is shown in Figs. 2 and 3 for $0.1 \mathrm{~mm}$ thick tungsten primary collimators. This collimator produces a very large tail of outscattered particles which will be lost on the aperture during the first half of a betatron oscillation. Most of these particles are intercepted by the downstream secondary collimators located $20^{\circ}$ and $37^{\circ}$ behind the primary ones. A supplementary collimator located immediately after these collimators, but from the other side of the beam, reduces losses behind the collimation section at injection by an additional factor of 14 . The supplementary collimator is located $2 \mathrm{~mm}$ farther from the beam than the secondary ones. Secondary and supplementary copper 

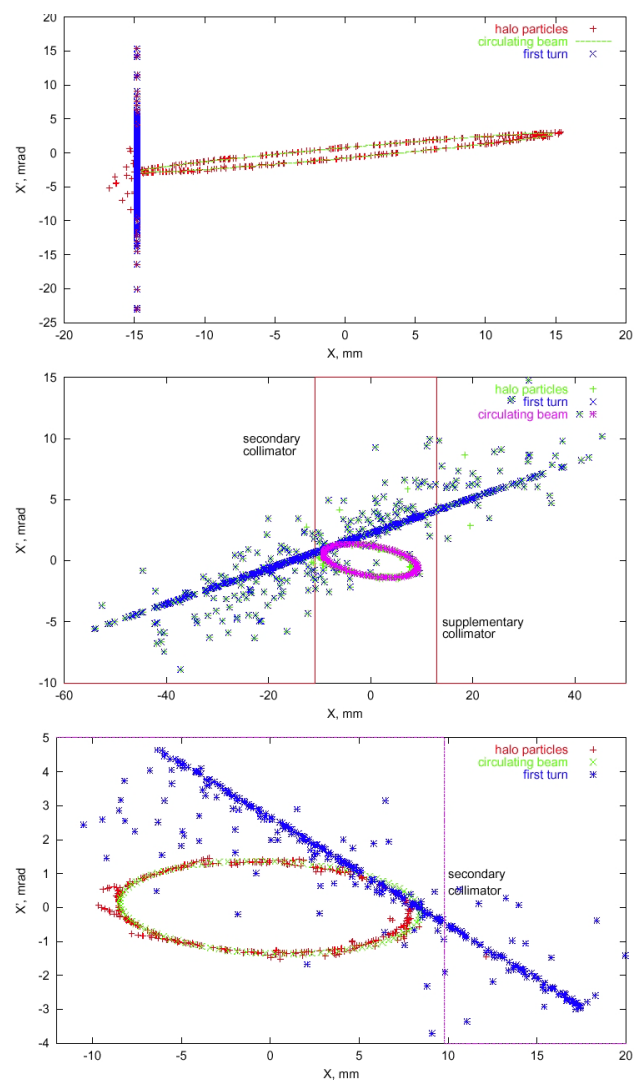

Figure 2: Horizontal phase space at injection in the primary collimator (top), secondary collimators SH1 (middle) and SH2 (bottom).

collimator length is $0.6 \mathrm{~m}$. Beam loss distributions at injection and at the top energy are shown in Fig. 4. This system localizes about $99 \%$ of beam loss in a $50 \mathrm{~m}$ long region. Beam loss in the rest of the machine is on average $0.1 \mathrm{~W} / \mathrm{m}$, with several peaks of $\sim 1 \mathrm{~W} / \mathrm{m}$. Note that the hands-on maintenance limits are $0.25 \mathrm{~W} / \mathrm{m}$ in the open long beam pipes and $3 \mathrm{~W} / \mathrm{m}$ in the magnets.

The loss rate in the magnets behind the primary collimators at injection is $\sim 100 \mathrm{~W} / \mathrm{m}$. It can be reduced to $1 \mathrm{~W} / \mathrm{m}$ if the primary collimator thickness is changed during the cycle from $0.003 \mathrm{~mm}$ to $0.1 \mathrm{~mm}$ for tungsten or from $0.15 \mathrm{~mm}$ to $5.4 \mathrm{~mm}$ for graphite. This can be done by rotation of primary collimator constructed as a disk of different thickness.

The mechanical design of the collimators will be similar to those already built and installed in the Tevatron for Collider Run II [3]. Those collimators (Fig. 5) consist of 2 pieces of copper welded together in an "L" configuration. The collimator assembly is bolted inside a stainless steel box. An expected power of $3.5 \mathrm{~kW}$ can be removed from a collimator by circulating low conductivity water through cooling channels on the outside of the collimator box. Detailed collimator mechanical design is underway.

Horizontal and vertical primary collimators consisting of $0.3 \mathrm{~mm}$ thickness carbon foils are now installed in the Booster. Preliminary observations of horizontal primary collimator interaction with beam have been made. The collimators are each electrically isolated to provide secondary
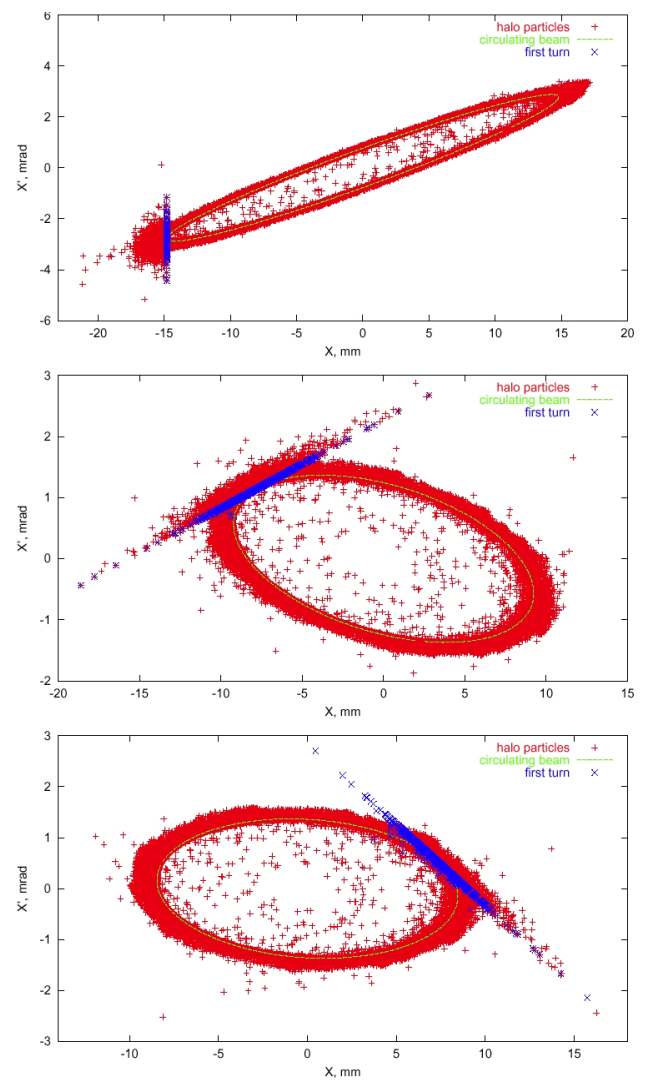

Figure 3: Horizontal phase space at the top energy in the primary collimator (top), secondary collimators SH1 (middle) and SH2 (bottom).

emission signals and instrumented at a 90 degree angle with photomultiplier detectors. Particles not captured in Booster
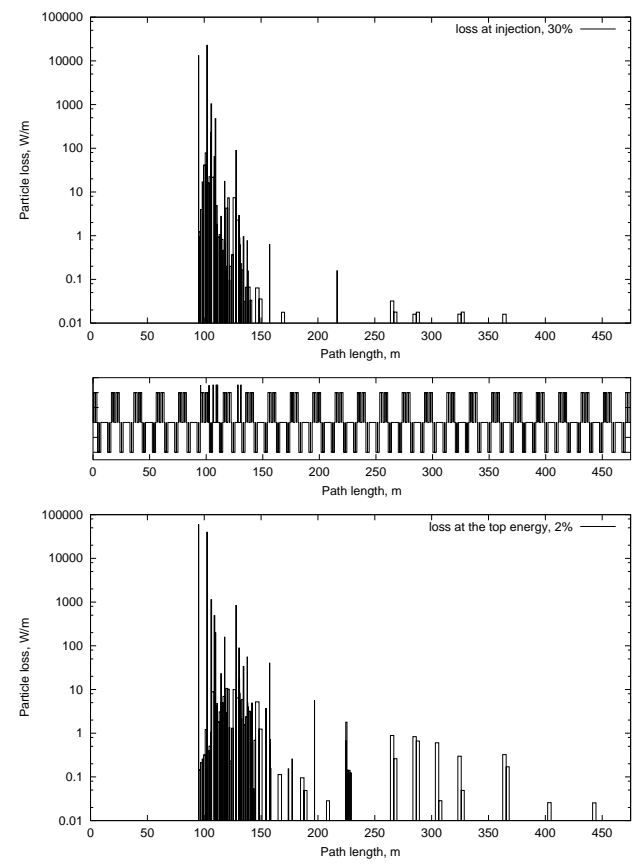

Figure 4: Beam loss distribution along the accelerator for $30 \%$ beam loss at injection (top) and for $2 \%$ beam loss at the top energy (bottom). 


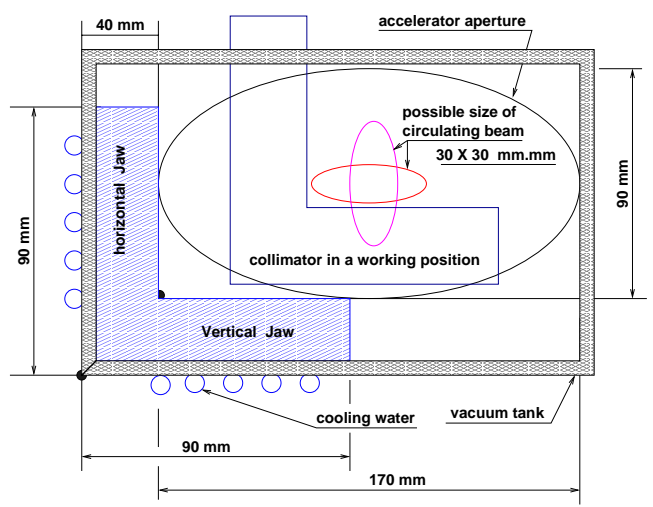

Figure 5: Secondary collimator cross section.

RF buckets are spilled from the machine during 400-700 microseconds after injection, as acceleration begins. This beam loss is clearly evident on the beam intensity signal in Fig. 6. With the collimator inserted into the machine aperture, but not so far as to be the cause of beam loss, the secondary emission and photomultiplier signals clearly show the collimator intercepting that beam spilling from the machine.

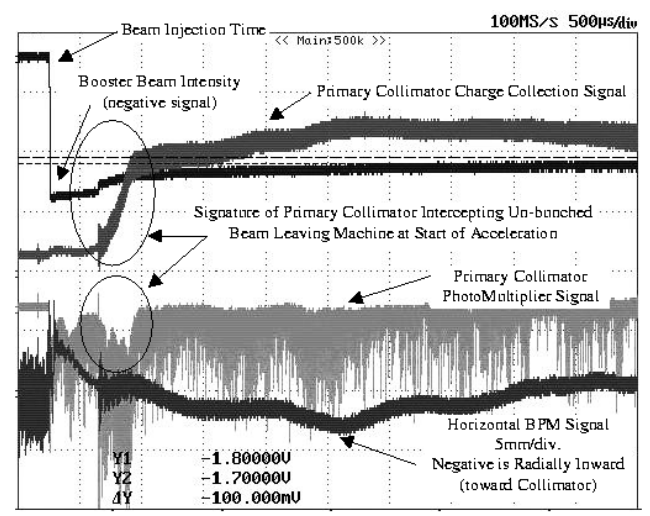

Figure 6: Horizontal primary collimator intercepting, without causing, beam loss.

\section{SHIELDING AND RELATED ISSUES}

As shown above, the total losses of $3.5 \mathrm{~kW}$ are spread in a $77 \%$ to $23 \%$ ratio between 6-th and 7-th straight sections. The MARS simulations in copper and inconel collimators for two positions of the beam relative to the collimator surface (Fig. 7) show that the collimators can sustain a continuous irradiation by a $8-\mathrm{GeV}$ beam which is at $\geq 1.5 \sigma$ from the collimator beam-side edge.

Dose and particle flux distributions in the hottest spots of the tunnel were calculated using a realistic source of particle loss derived from the STRUCT multi-turn simulations. It was found that the secondary collimators $\mathrm{SH} 1$ and $\mathrm{SH} 2$ should be embedded into iron shielding about $60 \mathrm{~cm}$ thick transversely (starting at $5 \mathrm{~cm}$ from the collimator outer surface) and extended about $60 \mathrm{~cm}$ downstream. Dose levels around such a shielding are quite acceptable, but it seems that it should be slightly longer. Fig. 8 shows hadron

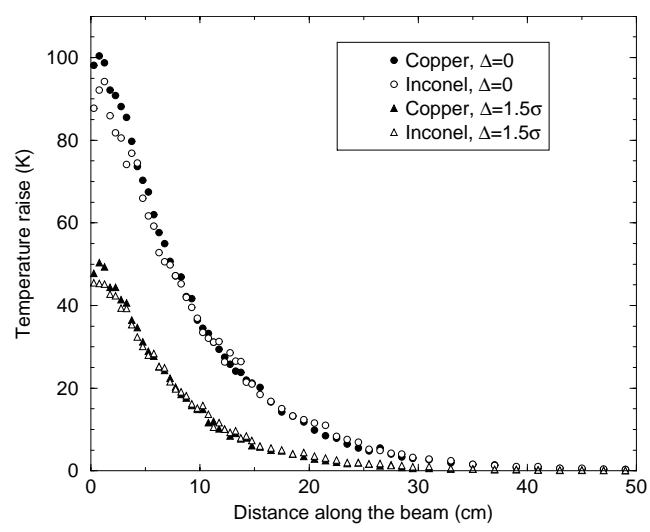

Figure 7: Instantaneous temperature rise along copper and inconel collimators for two positions $(\Delta)$ of the beam center relative to the collimator edge.

isofluxes $(\mathrm{E}>20 \mathrm{MeV})$ in the tunnel cross-section about $1.5 \mathrm{~m}$ from the entrance to $\mathrm{SH} 1$. One expects that extending the shielding by another 30 or $50 \mathrm{~cm}$ would reduce radiation levels as needed. Overall, despite of increased intensity of the Fermilab $8 \mathrm{GeV}$ Booster, beam loss and induced radiation effects can be controlled and reduced to the acceptable levels.

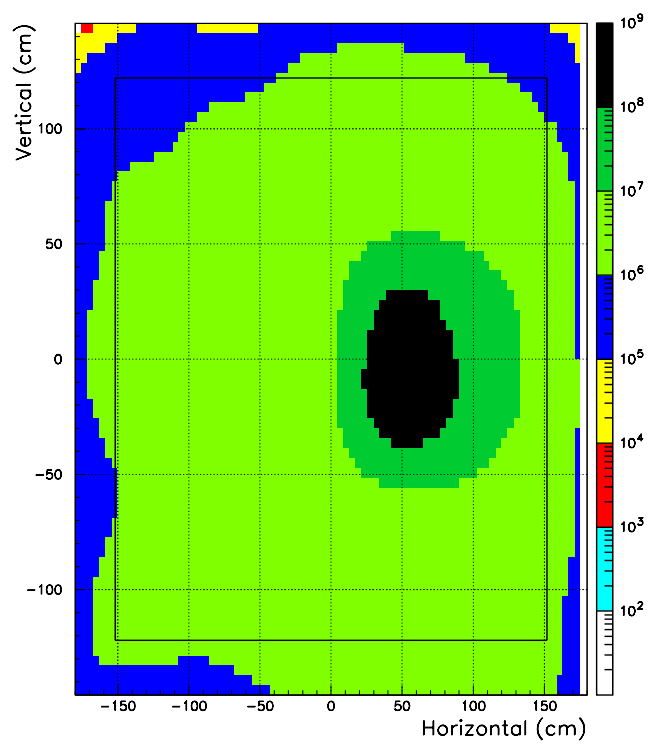

Figure 8: Hadron flux in $1 / \mathrm{cm}^{2} \mathrm{sec}$ after secondary collimator.

\section{REFERENCES}

[1] I. S. Baishev, A. I. Drozhdin and N. V. Mokhov, "STRUCT Program User's Reference Manual”, SSCL-MAN-0034 (1994); http://www-ap.fnal.gov/ drozhdin/.

[2] N. V. Mokhov, "The MARS Code System User's Guide", Fermilab-FN-628 (1995); N. V. Mokhov and O. E. Krivosheev, "MARS Code Status", Fermilab-Conf00/181 (2000). http://www-ap.fnal.gov/MARS/.

[3] M. Church, Proc. of the 7th ICFA Mini-Workshop on Beam Halo and Scraping, Lake Como, WI, Sept.1999, p.124. 